\title{
Darker eumelanic barn owls better withstand food depletion through resistance to food deprivation and lower appetite
}

\author{
Amélie Dreiss · Isabelle Henry • Charlène Ruppli • \\ Bettina Almasi · Alexandre Roulin
}

Received: 9 January 2010/ Accepted: 24 May 2010/Published online: 13 June 2010

(C) Springer-Verlag 2010

\begin{abstract}
The intensity of selection exerted on ornaments typically varies between environments. Reaction norms may help to identify the conditions under which ornamented individuals have a selective advantage over drab conspecifics. It has been recently hypothesized that in vertebrates eumelanin-based coloration reflects the ability to regulate the balance between energy intake and expenditure. We tested two predictions of this hypothesis in barn owl nestlings, namely that darker eumelanic individuals have a lower appetite and lose less weight when fooddeprived. We found that individuals fed ad libitum during $24 \mathrm{~h}$ consumed less food when their plumage was marked with larger black spots. When food-deprived for $24 \mathrm{~h}$ nestlings displaying larger black spots lost less weight. Thus, in the barn owl the degree of eumelanin-based coloration reflects the ability to withstand periods of food depletion through lower appetite and resistance to food restriction. Eumelanic coloration may therefore be associated with adaptations to environments where the risk of food depletion is high.
\end{abstract}

Keywords Appetite - Food depletion ·

Energy homeostasis · Melanin · Melanocortin

Communicated by Markku Orell.

A. Dreiss · I. Henry · C. Ruppli · A. Roulin $(\bowtie)$

Department of Ecology and Evolution, University of Lausanne,

Lausanne, Switzerland

e-mail: Alexandre.Roulin@unil.ch

B. Almasi

Swiss Ornithological Institute, 6204 Sempach, Switzerland

\section{Introduction}

Identifying the conditions under which body condition covaries with an ornament should bring useful information to an understanding of how selection is exerted on conspicuous ornamental traits. A positive relationship between the degree of ornamental exuberance and body condition can indicate that poor-quality individuals cannot invest resources to develop a conspicuous ornament at the expanse of body maintenance (Andersson 1994). In this case, an ornament can be considered as an honest signal of absolute quality because its expression is condition dependent. As the magnitude of a covariation between an ornament and body mass can vary, selection will favor ornamented individuals mainly in environments where selection is most intense. In cases where the sign of covariation varies between environments, we can conclude that differently ornamented individuals are adapted to alternate habitats, potentially indicating that different versions of an ornament reflect adaptations to local conditions (Bussière et al. 2008; Piault et al. 2009). This situation could occur if the expression of the ornament is condition dependent (van Doorn et al. 2009) but also if it is under strong genetic control.

Studying variation in the magnitude of a covariation between body condition and an ornament is particularly convenient in species displaying melanin-based color traits. In such species, inter-individual variation in coloration is often mainly assigned to genetic factors (e.g., Roulin 2004a; but see Fargallo et al. 2007) because the expression of melanic colorations can be insensitive to variation in the environment, a phenomenon referred to as "environmental canalization" (Flatt 2005; van Buskirk and Steiner 2009). While variation in the environment will affect body condition, each genotype will still produce a single color 
phenotype, implying that a change in the magnitude of covariation between body condition and coloration is driven by variation in body condition. Assessing environmentally mediated variation in such a covariation is of interest when identifying under which conditions some genotypes outperform others, genotypes being identified with the degree of melanin-based coloration.

In vertebrates, darker individuals have been proposed to be more resistant to various sources of stress and to more efficiently regulate the balance between energy intake and expenditure (Ducrest et al. 2008). Empirical studies indeed showed that the degree of melanic colorations is associated with energetic processes. For example, in the great tit (Parus major) and pied flycatcher (Ficedula hypoleuca) the degree of plumage darkness is positively correlated with oxygen consumption (Røskaft et al. 1986). We would thus predict that dark individuals better regulate body mass than paler conspecifics mainly in stressful environments. This would indicate that dark melanic individuals or species are particularly well adapted to harsh conditions. Accordingly, an observational study in the barn owl (Tyto alba) showed that dark breeding females are heavier than pale conspecifics in the evening but not in the morning (Roulin 2009). As owls consume food at night and fast during daylight hours, this observation suggests that some aspects of metabolism differ between dark and pale individuals.

In the present study, we carried out two experiments to test the hypothesis that darker eumelanic barn owls better regulate body mass than pale conspecifics. First, we fooddeprived nestlings for $24 \mathrm{~h}$ and measured body mass loss over this period of time. Second, we fed nestlings ad libitum and quantified the amount of mice consumed over $24 \mathrm{~h}$. Based on properties of the melanocortin system, which is involved in melanogenesis and energy homeostasis, we predict darker eumelanic owls to lose less weight when experimentally food-deprived and to have a lower appetite (Ducrest et al. 2008). Support for these predictions would indicate that a dark melanic coloration reveals the ability to withstand periods of food depletion through low appetite and resistance to food deprivation.

\section{Materials and methods}

\section{Model organism}

The barn owl is medium sized with adult females weighing between 264 and $515 \mathrm{~g}$ (mean $\pm \mathrm{SD} 367 \pm 1.5 \mathrm{~g}$ ) and adult males between 241 and $380 \mathrm{~g}$ (295 $\pm 1.6 \mathrm{~g})$. Two to 11 eggs per clutch are incubated for 32 days and hatch asynchronously every 2-3 days. Maximal growth occurs between 17 and 40 days of age, and before fledging at 56 days nestlings spontaneously lose weight. The species is particularly convenient for the manipulation of hunger levels because from 2 to 3 weeks of age onwards nestlings consume small mammals without maternal help not only at night but also during daylight hours as parents frequently store food (Roulin 2004b). Previous studies showed that in natural conditions 36-day-old nestlings eat on average 3.4 voles per $24 \mathrm{~h}$ (Roulin 2001) and in laboratory conditions mean \pm SD daily food intake was $67 \pm 17 \mathrm{~g}$ between 20 and 60 days of age (Durant and Handrich 1998). Barn owls are mostly monogamous with very little extra-pair paternity (one out of 211 offspring was not sired by the male that was feeding it, Roulin et al. 2004). Food deprivation over $24 \mathrm{~h}$ is not rare in natural conditions as during rainy nights parents have difficulty hunting.

Assessment of melanin-based plumage traits

Nestling and adult barn owls vary in both number and size of eumelanic black spots but also in pheomelanin-based coloration from dark reddish-brown to white. These traits are genetically correlated with darker reddish owls displaying on average more and larger eumelanic spots. The expression of melanin-based traits is strongly heritable and only weakly sensitive to environmental factors $\left(h^{2}=0.82\right.$; Roulin and Dijkstra 2003; Roulin et al. 2010). We measured plumage traits in 208 nestlings and in their parents (56 mothers and 52 fathers). A. Roulin compared pheomelanin-based coloration on the breast, belly, one flank and the underside of one wing with eight color chips ranging from I for reddish to VIII for white. As on each body part feathers are all similarly colored, we calculated a mean value to be used in the statistical analyses. Within a $60 \times 40-\mathrm{mm}$ frame placed on the same four body parts, eumelanic spots were counted and their diameter measured to the nearest $0.1 \mathrm{~mm}$. Mean number of spots and mean spot diameter were calculated and used in the statistical analyses. Assessing plumage traits is reliable with repeatability values varying between 0.84 and 0.93 (Roulin 1999, 2004c). We did not consider the intensity of spot darkness because spots are lighter colored when feathers are older implying that this parameter depends on the degree of feather abrasion, which is not the case with respect to number and size of spots.

\section{Experimental design}

The study was carried out in 2008 in western Switzerland in a free-ranging population of barn owls breeding in nestboxes. We cross-fostered approximately half of the hatchlings between pairs of nests to allocate genotypes randomly among the environments. The same number of nestlings was swapped between nests and hence brood size was left unchanged. Nestling position in the within-brood 
age hierarchy in the nests of origin and of rearing was not associated with nestling plumage traits (mixed-models, $P>0.10$ ). Within pairs of nests biological and foster parents did not resemble each other with respect to plumage traits (Pearson's correlations, all $P>0.15$ ), except that the number of spots displayed by biological and foster mothers were negatively correlated $(r=-0.33, n=47$, $P=0.022$ ). Among the 57 experimental nests hatching date was not correlated with plumage traits of biological parents $(r<0.20, P>0.14)$ except for males displaying small black spots that bred earlier in the season than males with larger spots $(r=0.34, n=53, P=0.012)$.

To record body mass change in nestlings under controlled conditions, from 12 June to 1 October we brought 208 nestlings from 57 origins to the laboratory for 3 nights. Nestling age (mean \pm SE $34 \pm 6$ days; range 18-51 days) was not correlated with plumage traits measured in their biological parents (Pearson's correlations on mean sibling values, $P>0.09$ ). Nestlings were brought to the laboratory in the afternoon at 1610 hours $( \pm 2 \mathrm{~h} 32 \mathrm{~min}$; SE) and their body mass measured to the nearest gram. Nestling body mass before the experiment was not significantly correlated with plumage traits measured in the nestlings themselves or in their biological parents (mixed-model analysis of covariance with nest of origin as random variable, controlling for nestling sex and age, $P>0.09$ ) but with nestling age (Pearson's correlation $r=0.62$, $P<0.0001)$. At their arrival nestlings were assigned to a food treatment for the night: they were either starved or offered food ad libitum, i.e., $130 \mathrm{~g}$ of laboratory mice which exceeds their daily food requirement. Remaining food was removed the next afternoon at 1600 hours and nestling body mass change over $24 \mathrm{~h}$ was determined. The food treatment was inverted the second night at 0000 hours (i.e., starved nestlings were fed ad libitum). Remaining food was also removed the following afternoon at 1600 hours and body mass change determined. On the first night we food-deprived 98 nestlings and fed the 110 other nestlings ad libitum. Plumage traits of nestlings that were food-deprived or fed ad libitum on the first night were similar (Student's $t$ test: color $t_{184}=0.76, P=0.45$; spot diameter $t_{184}=0.46, P=0.65$; number of spots $t_{184}=0.26, P=0.80$ ). At the end of the experiment (i.e., the morning following the third night), we brought nestlings back to their original nest-boxes, after having fed them ad libitum the third night. In the laboratory, nestlings were placed in a similar nest-box to the one where they were reared in natural conditions, but which was divided into two parts by a thin wooden wall pierced with five holes at the top. Each nestling was alone in one part of a nest-box while the other part was either empty $(n=20)$, occupied by a sibling $(n=84)$ or an unrelated individual raised in the same nest $(n=104)$.
Detailed observations on vocalizations produced by these nestlings (Roulin et al. 2009) showed that individuals behave as in natural conditions, indicating that conditions met in the laboratory were not too stressful. To further investigate the impact of keeping individuals in the laboratory, we measured baseline corticosterone levels (see Almasi et al. 2009 for details on the methods) in 20 nestlings just before bringing them in the laboratory and on average 2 days later in the laboratory just after the ad libitum food treatment was finished. This hormone is sensitive to various sources of stress (e.g., Jenni-Eiermann et al. 2008) and hence if nestlings are stressed to higher levels in the lab compared to the situation prevailing in their natural nest, baseline corticosterone levels are expected to be significantly higher in the lab. This was not the case (mean \pm SD baseline corticosterone level in the field was $5.57 \pm 4.2 \mathrm{ng} / \mathrm{ml}$ and in the laboratory $7.58 \pm 4.4 \mathrm{ng} / \mathrm{ml}$; paired $t$ test on log-transformed values $\left.t_{19}=1.24, P=0.23\right)$.

\section{Statistical procedure}

To assess the relationship between the degree of melaninbased coloration and body mass under contrasting feeding conditions (i.e., fed ad libitum vs. food-deprived during $24 \mathrm{~h}$ ) we performed a mixed-model ANOVA with body mass change over $24 \mathrm{~h}$ as the dependent variable. We included as random variables the nest of origin and the identity of each nestling nested in the nest of origin (since each individual appeared twice in the analysis, a first time when food-deprived and a second time when fed ad libitum). Covariates were nestling age, the three melaninbased plumage traits (pheomelanin-based coloration, spot diameter and spot number) measured in nestlings and in their two biological parents. We also introduced as cofactors nestling sex, food treatment, order of the food manipulation across the two experimental nights (i.e., starting with food-depriving individuals or by feeding them ad libitum) and nestling neighborhood in the laboratory nest-box (alone, in the presence of a sibling or of an unrelated but familiar individual raised in the same nest in the field). In a separate mixed-model ANOVA we introduced amount of mice consumed during $24 \mathrm{~h}$ in nestlings fed ad libitum. Nestling status (raised by the biological or foster parents), alone or in interaction did not account for any variation in body mass change and appetite. We thus removed this factor from the final models, which contained only significant effects and main effects involved in significant interactions. If we replace nestling age by initial body mass (the two variables are highly correlated, $r=0.62$ ), we obtain qualitatively similar results. Final models always presented a smaller Akaike information criterion than previous models containing non-significant 
terms. Assumptions for using parametric tests (homoscedastic and normal distributions of variables or residuals) were verified for each test. $P$ values smaller than 0.05 are considered significant.

\section{Results}

The final model testing whether nestling body mass change over $24 \mathrm{~h}$ is associated with spot diameter is given in Table 1. There were three major results from this analysis. First, nestlings lost less mass when their mother displayed larger black spots independently of the food treatment (Fig. 1). Second, the spot diameter of the father, alone or in interaction with other variables, did not explain any significant part of the variation. Third, there was a significant interaction between food treatment and nestling spot diameter. Nestlings displaying large eumelanic spots lost less body mass than those exhibiting smaller spots when food-deprived (mixed-model ANOVA with nest of origin as a random variable, $F_{1,126}=6.31, P=0.013$; Fig. 2) but not when fed ad libitum (similar model $F_{1,127}=1.66$,

Table 1 Final mixed-model ANOVA testing whether body mass change over $24 \mathrm{~h}$ in nestling barn owls is associated with the size of eumelanic spots measured in nestlings and in their biological parents

\begin{tabular}{lrrl}
\hline Source of variation & $d f$ & \multicolumn{2}{l}{$P$} \\
\hline Nestling pheomelanic coloration & 1,171 & 0.85 & 0.36 \\
Nestling spot number & 1,171 & 0.01 & 0.97 \\
Nestling spot diameter & 1,180 & 0.81 & $\mathbf{0 . 3 7}$ \\
Mother pheomelanic coloration & 1,180 & 2.00 & 0.16 \\
Mother spot number & 1,171 & 0.64 & 0.42 \\
Mother spot diameter & 1,180 & 6.82 & $\mathbf{0 . 0 0 9 8}$ \\
Father pheomelanic coloration & 1,171 & 1.95 & 0.16 \\
Father spot number & 1,171 & 0.61 & 0.44 \\
Father spot diameter & 1,171 & 0.01 & 0.96 \\
Nestling sex & 1,171 & 0.24 & 0.62 \\
Nestling age & 1,180 & 5.06 & $\mathbf{0 . 0 2 6}$ \\
Food manipulation & 1,180 & 176.36 & $<\mathbf{0 . 0 0 0 1}$ \\
Order of food manipulation & 1,180 & 233.11 & $<\mathbf{0 . 0 0 0 1}$ \\
Food manipulation $\times$ order of food & 1,180 & 91.49 & $<\mathbf{0 . 0 0 0 1}$ \\
$\quad$ manipulation & & & \\
Food manipulation $\times$ nestling spot & 1,180 & 6.95 & $\mathbf{0 . 0 0 9 1}$ \\
$\quad$ diameter & & & \\
\hline
\end{tabular}

Nest of origin was incorporated as a random variable as well as nestling identity nested within the nest of origin. Independent variables were food manipulation (over $24 \mathrm{~h}$ individuals were either fed ad libitum or food-deprived), and the order of this manipulation (in approximately half of the cases individuals were first fed ad libitum and others were first food-deprived). Non-significant interactions were removed one after the other starting with the least significant ones

$P$ values of the final model are in bold and non-significant $P$ values of initial models in plain

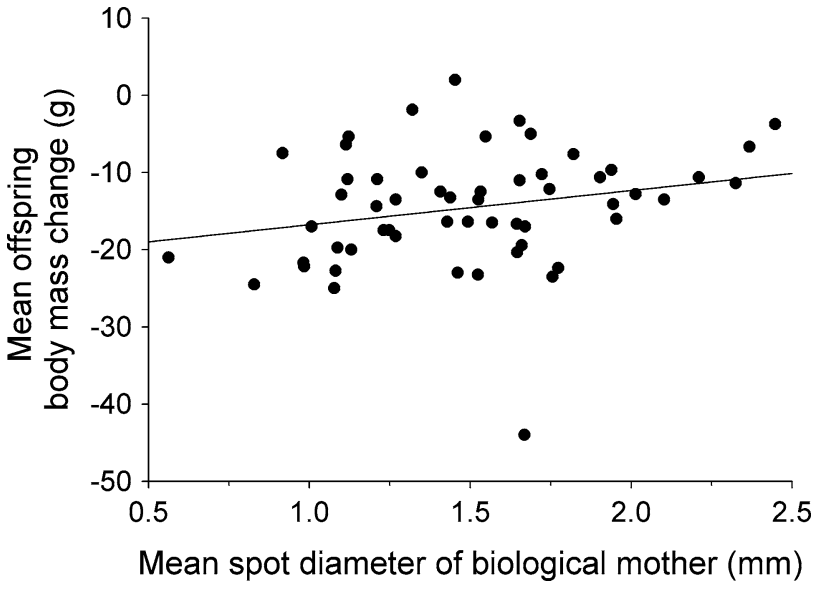

Fig. 1 Mean body mass change (in g) in nestling barn owls over $24 \mathrm{~h}$ in relation to the size of eumelanic spots measured in their biological mother. A mean sibling value was calculated so that each mother appears only once in this figure (Pearson's correlation is $r=0.38$, $n=43, P=0.01)$. The regression line is shown

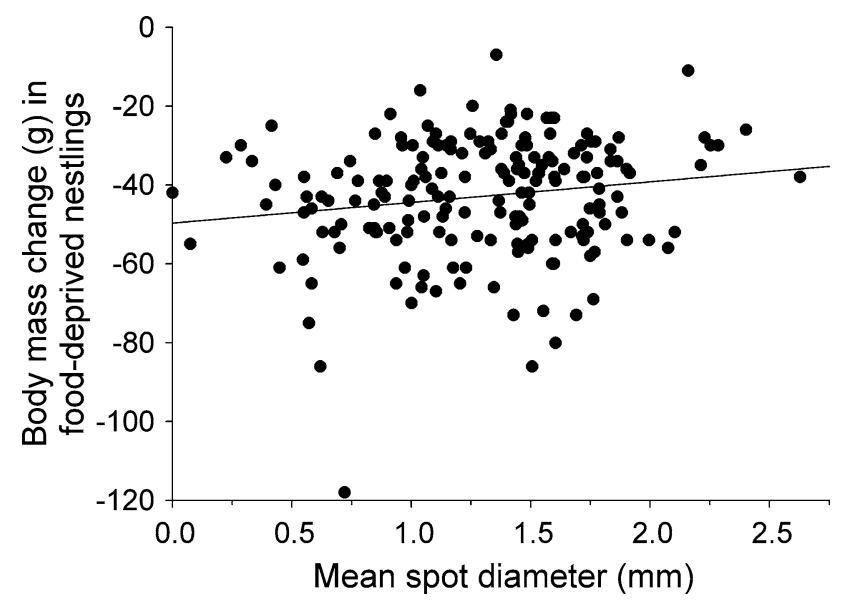

Fig. 2 Body mass change (in g) in nestling barn owls that were fooddeprived over $24 \mathrm{~h}$ in relation to the size of their eumelanic spots. The regression line is shown

$P=0.20)$. The lack of relationship between nestling spot diameter and nestling body mass could be due to differential appetite. Accordingly, when nestlings were fed ad libitum, individuals displaying large black spots consumed fewer mice than individuals displaying smaller spots (Table 2; Fig. 3a). Whitish individuals had less appetite than reddish ones, but the relationship was relatively weak (Table 2; Fig. 3b).

\section{Discussion}

Under laboratory conditions food-deprived nestling barn owls that displayed larger black spots lost less weight and had a lower appetite. These results shed new light on the 
Table 2 Final mixed-model ANOVA testing whether appetite (expressed in $\mathrm{g}$ mice eaten over $24 \mathrm{~h}$ ) in nestling barn owls fed ad libitum is associated with the size of eumelanic spots measured in nestlings and in their biological parents

\begin{tabular}{llcl}
\hline Source of variation & $d f$ & $F$ & $P$ \\
\hline Nestling pheomelanic coloration & 1,126 & 5.29 & $\mathbf{0 . 0 2 3}$ \\
Nestling spot number & 1,117 & 1.46 & 0.23 \\
Nestling spot diameter & 1,126 & 7.52 & $\mathbf{0 . 0 0 7}$ \\
Mother pheomelanic coloration & 1,117 & 0.90 & 0.34 \\
Mother spot number & 1,117 & 0.18 & 0.67 \\
Mother spot diameter & 1,117 & 0.49 & 0.49 \\
Father pheomelanic coloration & 1,117 & 0.62 & 0.43 \\
Father spot number & 1,117 & 0.03 & 0.87 \\
Father spot diameter & 1,117 & 0.86 & 0.35 \\
Nestling sex & 1,117 & 0.27 & 0.61 \\
Nestling age & 1,117 & 0.01 & 0.99 \\
Nestling body mass before feeding & 1,126 & 4.69 & $\mathbf{0 . 0 3 2}$ \\
Order of food manipulation & 1,126 & 35.80 & $<\mathbf{0 . 0 0 0 1}$ \\
\hline
\end{tabular}

Nest of origin was incorporated as a random variable. Independent variables were melanin-based plumage traits of nestling and their biological parents, nestling age, body mass before the feeding treatment was applied, sex and the order of food manipulation (in approximately half of the cases individuals were first fed ad libitum and others were first food-deprived). Non-significant interactions were removed one after the other starting with the least significant ones

$P$ values of the final model are in bold and non-significant results of initial model in plain

physiological adaptations associated with melanin-based color traits. A number of recent studies have indeed proposed that such colorful traits could indicate various physiological properties including for instance resistance to oxidative stress and parasites as well as energy homeostasis (Ducrest et al. 2008). These studies were based on knowledge of the physico-chemical effects of melanin pigments (Mackintosh 2001; McGraw 2005) or of physiological effects of biochemical molecules involved in melanogenesis. Interestingly, our results are consistent with pleiotropic effects of the melanocortin system suggesting that mutations located in the proopiomelanocortin gene or differential expression of this gene are responsible for the link between melanin-based coloration and energy homeostasis (Ducrest et al. 2008).

Our study has several implications for an understanding of the potential adaptive function of melanin-based color traits. Several studies suggest that darker eumelanic individuals are adapted to stressful conditions. For example, darker feral pigeons (Columba livia) better survived after the Chernobyl catastrophe (Johnston and Janiga 1995), offspring of darker melanic Alpine swift (Apus melba) fathers grew more rapidly when brood size was experimentally enlarged but not when experimentally reduced (Roulin et al. 2008), in siskins (Carduelis spinus) darker individuals were less susceptible to stressful laboratory conditions as measured by metabolic rate (Senar et al. 2000), and in nestling common buzzards (Buteo buteo) intensity of infection with Leucocytozoon endoparasites decreased with melanization (Chakarov et al. 2008). In the barn owl, females displaying larger eumelanic spots gave birth to offspring that were more resistant to ectoparasites, produced more antibodies towards a vaccine and were developmentally more stable (Roulin 2004c), male barn owls displaying larger black spots were less sensitive to an experimental increase in corticosterone level (Almasi et al. 2008) and offspring born from heavily spotted mothers were better able to cope with a rise in corticosterone levels due to stressful situations (Almasi et al. 2010). The present study provides further information on the ability to cope with food depletion. Darker eumelanic individuals better dealt with experimental food deprivation and had a lower appetite suggesting that they need less food to sustain metabolism. Our experiment should be repeated under natural conditions to determine whether the relationships between melanin-based coloration and energetic processes are not specific to stressful conditions such as those probably met under laboratory conditions. The observation that melanin-based coloration co-varied significantly with aspects of body condition only after we manipulated food supply is consistent with the claim that the degree of pigmentation signals quality only under specific conditions
Fig. 3 Amount of mice (in g) nestling barn owls consumed in $24 \mathrm{~h}$ in relation to the size of their eumelanic spots (a) and pheomelanin-based coloration (b)
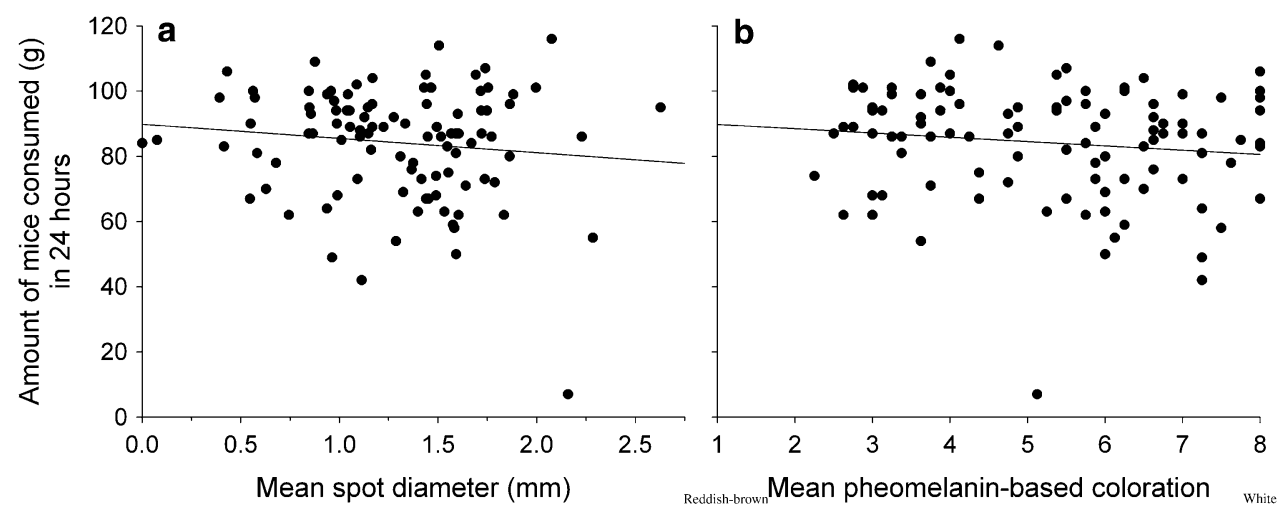
(Roulin 2009). Therefore, selection on melanin-based color traits is context dependent [see also Gonzales et al. (1999) for another example].

In the present experimental study and the previous observational study carried out in barn owls (Roulin 2009), the balance between energy intake and expenditure was mainly associated with the degree of eumelanin- and to a low extent with pheomelanin-based coloration. This contrasts with a similar experimental study we recently carried out in the tawny owl (Strix aluco), a species that varies in the degree of pheomelanin-based coloration. In the laboratory, food-deprived tawny owl nestlings lost less mass when their biological mother was pale rather than dark reddish-brown (Piault et al. 2009). This result is interesting because the intensity of melanin pigmentation is positively correlated with the ability to cope with food depletion with respect to eumelanic coloration in the barn owl but negatively with respect to pheomelanic coloration in the tawny owl. From a proximate point of view, this suggests that a molecule that triggers the production of eumelanic pigments binds to other receptors responsible for energy homeostasis which are also sensitive to an antagonistic molecule that triggers the production of pheomelanic pigments (Ducrest et al. 2008). From an ultimate point of view, this may indicate that pheomelanic and eumelanic colorations may signal similar phenotypic attributes but in opposite directions.

To conclude, if the degree of melanin-based coloration is associated with the ability to deal with poor food conditions, we could predict that dark eumelanic color traits are more prevalent in populations or in species that face a higher risk of food shortage. This is a stimulating avenue of research, which has not yet been considered.

Acknowledgments The Swiss National Science Foundation supported this study financially (Grant no. PPOOA-102913 to A. R.). The experiments were approved by the veterinary services of Canton de Vaud (licence no. 2109.0). We thank Oliver Krueger and an anonymous referee for helpful comments on an earlier version of the paper.

\section{References}

Almasi B, Roulin A, Jenni-Eiermann S, Jenni L (2008) Parental investment and its sensitivity to corticosterone is linked to melanin-based coloration in barn owls. Horm Behav 54:217223. doi:10.1016/j.yhbeh.2008.02.021

Almasi B, Roulin A, Jenni-Eiermann S, Breuner CW, Jenni L (2009) Regulation of free corticosterone and CGG capacity under different environmental conditions in altricial nestlings. Gen Comp Endocrinol 164:117-124. doi:10.1016/j.ygcen.2009.05.011

Almasi B, Jenni L, Jenni-Eiermann S, Roulin A (2010) Regulation of stress-response is heritable and functionally linked to melaninbased coloration. J Evol Biol 23:987-996. doi:10.1111/j.14209101.2010.01969.x

Andersson M (1994) Sexual selection. Princeton University Press, Princeton
Bussière LF, Hunt J, Stölting KN, Jennions MD, Brooks R (2008) Mate choice for genetic quality when environments vary: suggestions for empirical progress. Genetica 134:69-78. doi: 10.1007/s10709-007-9220-z

Chakarov N, Boerner M, Krüger O (2008) Fitness in common buzzards at the cross-point of opposite melanin-parasite interactions. Funct Ecol 22:1062-1069. doi:10.1111/j.1365-2435.2008. 01460.x

Ducrest A-L, Keller L, Roulin A (2008) Pleiotropy in the melanocortin system coloration and behavioural syndromes. Trends Ecol Evol 23:502-510. doi:10.1016/j.tree.2008.06.001

Durant JM, Handrich Y (1998) Growth and food requirement flexibility in captive chicks of the European barn owl (Tyto alba). J Zool 245:137-145. doi:10.1111/j.1469-7998.1998.tb00083.x

Fargallo JA, Laaksonen T, Korpimaki E, Wakamatsu K (2007) A melanin-based trait reflects environmental growth conditions of nestling male Eurasian kestrels. Evol Ecol 21:157-171. doi: 10.1007/s10682-006-0020-1

Flatt T (2005) The evolutionary genetics of canalization. Q Rev Biol 80:287-316. doi:10.1086/432265

Gonzales G, Sorci G, de Lope F (1999) Seasonal variation in the relationship between cellular immune response and badge size in male house sparrows (Passer domesticus). Behav Ecol Sociobiol 46:117-122. doi:10.1007/s002650050600

Jenni-Eiermann S, Glaus E, Gruebler M, Schwabl H, Jenni L (2008) Glucocorticoid response to food availability in breeding barn swallows (Hirundo rustica). Gen Comp Endocrinol 155:558565. doi:10.1016/j.ygcen.2007.08.011

Johnston RF, Janiga M (1995) Feral pigeon. Oxford University Press, Oxford

Mackintosh JA (2001) The antimicrobial properties of melanocytes melanosomes and melanin and the evolution of black skin. J Theor Biol 211:101-113. doi:10.1006/jtbi.2001.2331

McGraw KJ (2005) The antioxidant function of many animal pigments: are there consistent health benefits of sexually selected colorants? Anim Behav 69:757-764. doi:10.1016/j.anbehav. 2004.06.022

Piault R, Gasparini J, Bize P, Jenni-Eiermann S, Roulin A (2009) Pheomelanin-based coloration and the ability to cope with variation in food supply and parasitism. Am Nat 174:548-556. doi: $10.1086 / 605374$

Røskaft E, Järvi T, Bakken M, Bech C, Reinertsen RE (1986) The relationship between social status and resting metabolic rate in great tits (Parus major) and pied flycatchers (Ficedula hypoleuca). Anim Behav 34:838-842. doi:10.1016/S0003-3472(86) 80069-0

Roulin A (1999) Nonrandom pairing by male barn owls Tyto alba with respect to a female plumage trait. Behav Ecol 10:688-695. doi:10.1093/beheco/10.6.688

Roulin A (2001) Food supply differentially affects sibling negotiation and competition in the barn owl (Tyto alba). Behav Ecol Sociobiol 49:514-519. doi:10.1007/s002650100322

Roulin A (2004a) The evolution maintenance and adaptive function of genetic color polymorphism in birds. Biol Rev 79:815-848. doi: $10.1017 /$ S1464793104006487

Roulin A (2004b) Function of food stores in bird nests: observations and experiments in the barn owl Tyto alba. Ardea 92:69-78

Roulin A (2004c) Proximate basis of the covariation between a melanin-based female ornament and offspring quality. Oecologia 140:668-675. doi:10.1007/s00442-004-1636-x

Roulin A (2009) Covariation between eumelanic pigmentation and body mass only under specific conditions. Naturwissenschaften 96:375-382. doi:10.1007/s00114-008-0489-2

Roulin A, Dijkstra C (2003) Genetic, environmental components of variation in eumelanin and phaeomelanin sex-traits in the barn owl. Heredity 90:359-364. doi:10.1038/sj.hdy.6800260 
Roulin A, Müller W, Sasvári L, Dijkstra C, Ducrest A-L, Riols C, Wink M, Lubjuhn T (2004) Extra-pair paternity testes size and testosterone level in relation to color polymorphism in the barn owl Tyto alba. J Avian Biol 35:492-500. doi:10.1111/j.09088857.2004.03294.x

Roulin A, Gasparini J, Bize P, Ritschard M, Richner H (2008) Melanin-based colorations signal strategies to cope with poor and rich environments. Behav Ecol Sociobiol 62:507-519. doi: 10.1007/s00265-007-0475-2

Roulin A, Dreiss A, Fioravanti C, Bize P (2009) Vocal sib-sib interactions: how siblings adjust signaling level to each other. Anim Behav 77:717-725. doi:10.1016/j.anbehav.2008.12.004

Roulin A, Altwegg R, Jensen H, Steinsland I, Schaub M (2010) Sexdependent selection on an autosomal melanic female ornament promotes the evolution of sex ratio bias. Ecol Lett 13:616-626. doi:10.1111/j.1461-0248.2010.01459.x

Senar JC, Polo V, Uribe F, Camerino M (2000) Status signalling metabolic rate and body mass in the siskin: the cost of being subordinate. Anim Behav 59:103-110. doi:10.1006/anbe.1999. 1281

Van Buskirk J, Steiner UK (2009) The fitness costs of developmental canalization and plasticity. J Evol Biol 22:852-860. doi:10.1111/ j.1420-9101.2009.01685.x

Van Doorn GS, Edelaar P, Weissing FJ (2009) On the origin of species by natural and sexual selection. Science 326:1704-1707. doi:10.1126/science.1181661 